\title{
Customised pressure profiles of made-to-measure sports compression garments
}

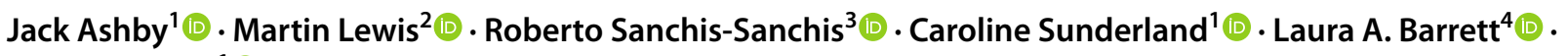 \\ John G. Morris ${ }^{1}$ (i)
}

Accepted: 4 May 2021 / Published online: 22 May 2021

(c) The Author(s) 2021

\begin{abstract}
The purpose of this study was to make made-to-measure compression garments that elicit pressures within and below clinical standards. The study also examined whether pressures and gradients can be replicated within and between participants' legs, and between separate compression garment conditions. Ten males volunteered to participate. Based on three-dimensional scans of the participants' lower body, three different made-to-measure garments were manufactured: control, symmetrical and asymmetrical. Garment pressures were assessed from the malleolus to the gluteal fold using a pressure monitoring device. A root mean squared difference analysis was used to calculate the in vivo linear graduation parameters. Linear regression showed that peak pressure at the ankle in the left and right leg were: control garment, $13.5 \pm 2.3$ and $12.9 \pm 2.6$; asymmetrical garment, $12.7 \pm 2.5$ and $26.3 \pm 3.4$; symmetrical garment, $27.7 \pm 2.2$ and $27.5 \pm 1.6$ (all mmHg, mean \pm standard deviation). Pressure reduction from the ankle to the gluteal fold in the left and right leg were: control, 8.9 \pm 3.5 and 7.4 \pm 3.0 ; asymmetrical, $7.8 \pm 3.9$ and 21.9 \pm 3.2 ; symmetrical, $25.0 \pm 4.1$ and $22.3 \pm 3.6$ (all $\mathrm{mmHg}$, mean \pm standard deviation). Made-tomeasure compression garments can be made to elicit pressures within and below clinical standards, and to elicit equivalent pressures and gradients in different participants.
\end{abstract}

\section{Introduction}

Compression garments are worn to apply an external, mechanical pressure on the surface of the body, which may compress and support underlying tissues and have been shown to reduce muscle oscillation during exercise [1, 2]. In clinical practice, guidelines have been developed to ensure appropriate prescription of compression garment pressures for specific conditions. However, it should be noted that agreed pressure guidelines do not necessarily result in the same classifications in all countries; for example, in the UK, France and Germany, specific compression

Jack Ashby

jack.ashby@ntu.ac.uk

1 Department of Sport Science, Nottingham Trent University, Nottingham, UK

2 Department of Human Sciences, University of Derby, Derby, UK

3 Department of Physical Education and Sports, University of Valencia, Valencia, Spain

4 School of Sport, Exercise and Health Sciences, Loughborough University, Loughborough, UK garment pressures correspond to different classifications [3]. In the UK, the guidelines have three pressure classifications (BS-6612; 1985): Classes one (14-17 $\mathrm{mmHg})$, two (18-24 mmHg) and three $(25-35 \mathrm{mmHg})$. Wearing compression garments is common in sporting environments $[4,5]$. Some manufacturers claim that their garments elicit 'graduated compression'. Such claims of graduated compression implies that a garment elicits high pressures at the distal end, with the pressure gradually reducing towards the proximal end, which may improve venous flow and return [6]. Some research has found positive effects of wearing compression garments on exercise performance, or during recovery from exercise [7-12]. However, other research has not been able to demonstrate such effects [13-18]. Consequently, with such equivocal research findings, it is unknown whether compression garments aid exercise performance and recovery.

A factor that may explain the equivocal findings in the sport-related research literature is that many studies do not measure the pressure elicited by the compression garment, often reporting only manufacturer-estimated values typically taken from standardised wooden-leg models [19]. The inadequate quantification of between-human 
differences in leg geometry, and the different stiffness characteristics of leg tissues, such as bone, tendon and muscle, probably contribute to a limited understanding of the actual in vivo pressures elicited by compression garments. The ambiguity in the results of sport-related research involving compression garments is therefore perhaps unsurprising. If pressure is not measured, then linking the pressure profile elicited by a compression garment with any associated performance changes and physiological adaptations is impossible.

Commercially available compression garments are required to fit individuals whose body morphologies are unknown to the manufacturer, thus, generalized sizing systems are typically applied [1]. Such compression garments are typically available in five alphanumerical sizes, i.e. extra small, small, medium, large and extra large. This lack of customization could lead to garments that fit poorly. Therefore, if the pressures applied by commercially available compression garments were measured, these pressures could vary between individuals. Indeed, Brophy-Williams et al. [20] found the pressure elicited by compression garments to be affected by sizing. Furthermore, it was found that by wearing commercially available compression garments and using manufacturer recommended sizing, the measured pressures varied between individuals even if individuals were fitted within the same sizing category. As such, if a compression garment fits poorly there could be differences in pressure profiles experienced by participants. The requirement for a compression garment to provide the same fit between legs is also of importance. It is common to have size and shape differences between dominant and non-dominant legs. Rauter et al. [21] showed knee and calf circumferences to differ between left and right legs in young, male road cyclists. Such leg asymmetry could result in commercially available compression garments eliciting more pressure on one leg than the other, as well as providing an inconsistent fit between participants. To ensure robust research study design with sufficient reliability and validity, it may be beneficial for participants to wear made-to-measure compression garments, to allow all participants to experience equivalent pressure profiles. However, whether made-to-measure compression garments can be manufactured to ensure similar pressure profiles across individuals is unknown.

This study had three aims, first, to examine if it was possible to make a made-to-measure compression garment that elicits graduated pressures that fit within clinical pressure standards and a control compression garment with pressures below clinical standards. It also aimed to examine whether pressures and gradients can be replicated within and between participants' legs and for separate compression garment conditions. Finally, it aimed to examine made-to-measure compression garment fit between conditions and between participants' legs.

\section{Methods}

\subsection{Participants}

Ten healthy, recreational male runners (age $24.3 \pm 4.6$ years, stature $181.5 \pm 1.8 \mathrm{~cm}$, body mass $75.7 \pm 3.8 \mathrm{~kg}$, mean \pm standard deviation) volunteered and provided informed consent to participate in the study. All participants completed a health screen questionnaire before involvement in the study, to ensure they had no medical or other conditions that would have prevented them from taking part. Participants with medical conditions or injuries were excluded from the study. All participants refrained from strenuous exercise $24 \mathrm{~h}$ before each trial and refrained from caffeine on the day of a trial. The study was approved by a University Ethics Committee, (Nottingham Trent University Ethical Committee Application for Human Biological Investigation reference number: 560).

\subsection{Experimental design}

Participants visited the laboratory four times. The first visit was a familiarisation trial, which consisted of a baseline three-dimensional (3D) scan that was used to support the manufacture of made-to-measure compression garments for each participant. Each experimental trial consisted of wearing a different lower body compression garment whilst pressure profiles, defined as the pressure and pressure gradient from the distal to the proximal end of both legs, were measured. The compression garment conditions were: (1) control, (2) symmetrical and (3) asymmetrical (detailed in Sect. 2.3).

\subsection{Compression garments and 3D scan}

The study used made-to-measure, full leg compression tights (Kurio 3D Compression Ltd, Nottingham, UK) and were fitted from the malleolus to the iliac crest. Within each trial, the compression garment used differed in pressure and graduation of pressure. The pressure profile of each garment was implemented into the garment using a specifically designed software programme developed by the company. The compression garments were made using a composite of Elastane (22\%) and Nylon (78\%), in two sections as left and right legs with a seam up the centre line. The properties of the material were determined by the companies' in-house testing processes. An assessment of the material was made following the standard for evaluating the 'Determination of the elasticity of fabrics' (BS EN 14704-1:2005) then further evaluated against in vivo measurements of pressure obtained from individuals outside of the study population $(n=30)$ 
to establish the relationship between material reductions, body geometry and elicited pressure. The measured properties were then used to determine the material size reduction required to generate intended clinical and non-clinical pressures for all garments according to Laplace's Law [22]. The elastic material used for the garment facilitates dressing, such that the garment can be stretched over various joint structures. Furthermore, this stretching ensures that the garment sits on the appropriate surface of the limb without slipping. The control garment was designed to elicit pressure below clinical standards $(<14 \mathrm{mmHg})$ with no pressure gradient. The symmetrical garment was designed to elicit pressure within clinical standards (14-35 $\mathrm{mmHg}$ ) and to include a linear pressure gradient from distal to proximal (graduated compression). The asymmetrical garment was designed to elicit control conditions in the left leg and graduated compression in the right. The pressure classifications used in this study corresponded to UK compression standards (BS-6612; 1985): Classes one (14-17 mmHg), two (18-24 mmHg) and three $(25-35 \mathrm{mmHg})$.

To develop the made-to-measure compression garments, an Artec Eva 3D scanner (Artec Group, Luxembourg, Luxembourg) captured a lower-body 3D scan of each participant whilst they slowly rotated on a turntable, standing with their legs shoulder width apart. Scans were processed using Artec Studio 13 software (Artec Group, Luxembourg, Luxembourg) and exported to a custom-built programme (Kurio 3D Compression LTD), where identical garment resizing parameters were used for each participant to produce material templates that elicited the required pressures in the control, symmetrical and asymmetrical garment conditions.

\subsection{Pressure profile assessment}

The pressure profiles of the compression garments were assessed using a Kikuhime pressure-monitoring device (MediGroup, Melbourne, Australia). Previous research has assessed the accuracy and reliability of the pressure monitoring device using a water column reference method (typical error of measurement $= \pm 1 \mathrm{mmHg}$ ) [23]. Pressure elicited by the garments was measured at multiple sites on the midline of the posterior surface of each leg. The location of the pressure sensor measurement sites was acquired simultaneously with pressure measurements using a thirteen-camera 3D motion capture system (Qualisys AB, Göteborg, Sweden) sampling at $100 \mathrm{~Hz}$. Eight reflective markers were applied to the lower body limbs, using bi-adhesive tape, to represent the line of the leg. Four markers were placed on each leg at the following landmarks: (1) the lateral malleolus (ankle); (2) the lateral femoral condyle (knee); (3) the greater trochanter; and (4) the iliac crest. Before the pressure profile assessment participants would have been standing for twenty minutes. During the pressure profile assessment, participants

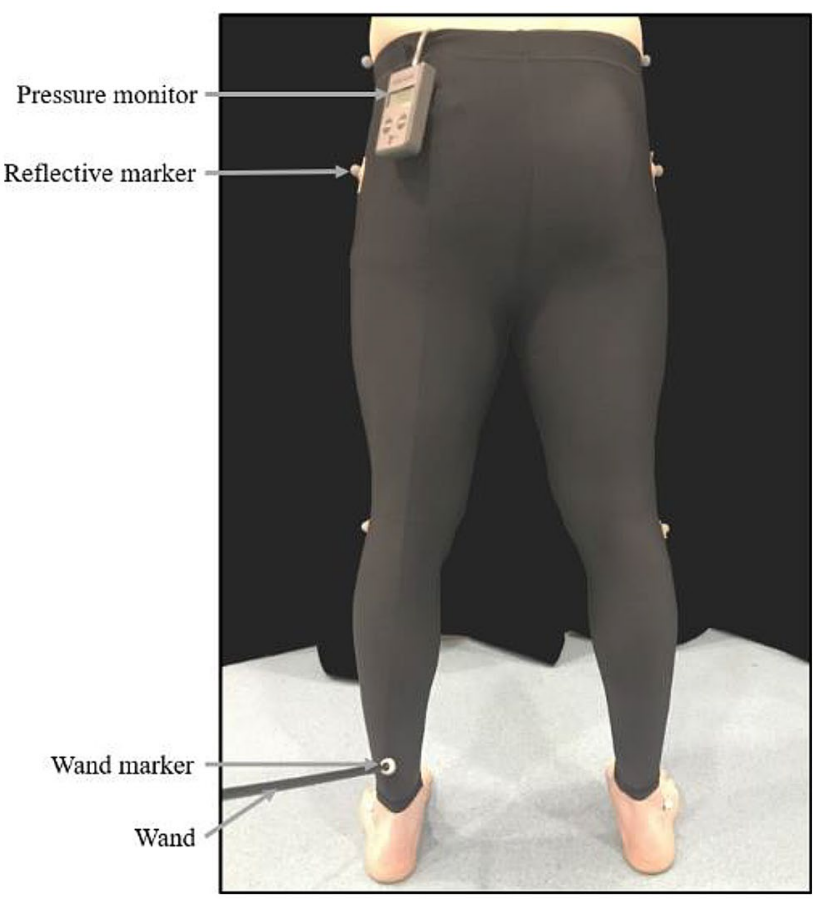

Fig. 1 Participant wearing the compression garment during the pressure profile assessment with the 'reflective markers' applied to define leg length, the 'pressure monitor' in place to measure pressure elicited by the garment (distal to proximal), the 'wand' and the 'wand marker' applied before each pressure measurement to reference the measurement location relative to leg length

stood with their legs shoulder width apart with their arms crossed over their chest. Participants were instructed to stand still and to keep their musculature relaxed during the pressure measurement. The pressure monitoring device was placed between the garment and skin interface and repeatedly relocated by pulling the pressure monitoring device up the limb for each measurement. Pressure measurements were collected at about $5 \mathrm{~cm}$ increments up the posterior surface of both limbs from the malleolus. To obtain a precise location for the pressure measurements, a reflective wand marker was briefly placed on the pressure measurement site before reading the pressure (see Fig. 1). The pressure profile assessment of both limbs lasted about eight minutes.

\subsection{Statistical analysis}

A root mean squared (RMS) difference analysis was used to calculate the in vivo (worn) linear graduation parameters of peak pressure and graduation. This analysis was used to assess differences of peak pressure and pressure gradient between conditions and between a participants' legs, to determine how well the garments fitted at both group and individual level. For this approach, individual participants' pressure data for each leg were fitted with the equation of a

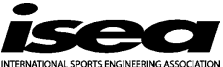


straight line (see Eq. 1) to identify the two parameters which minimised the difference between measured and predicted pressures using a simulated annealing algorithm [24]. The two parameters identified were peak pressure at the ankle malleolus $\left(p_{\max }\right)$ and the pressure gradient $\left(\Delta_{p}\right)$. The predicted pressure corresponded to the line of best fit generated by the algorithm.

A straight line was fitted to the data using

$P=\Delta_{p} x+p_{\max }$

where $p_{\max }$ is the peak pressure at the ankle, $\Delta_{p}$ is the pressure gradient (the reduction in pressure between the ankle malleolus and the greater trochanter) and $x$ is the percentage of leg length.

The parameters and RMS values were used to analyse garment fit between participants within a garment condition. When calculating RMS at participant limb level, the fitting of individual limb pressure measurements was made by minimising a conventional RMS, squaring each pressure measurement, then calculating their mean and square root (see Eq. 2).

The difference between a participants' measured pressure and the predicated pressure for the same measurement location was calculated using

$\mathrm{RMS}_{\text {ind }}=\sqrt{\frac{\sum_{i=1}^{n}\left(P a_{i}-P b_{i}\right)^{2}}{n}}$,

where $P a_{i}$ is the participants' measured pressure, $P b_{i}$ is the participants' model calculated pressure and $n$ is the total number of pressure measurements.

At group level, rather than square each participants' difference between measured and model calculated pressure, we instead adopted an approach of calculating the mean difference between measured and model calculated pressures for a participant and then calculated the sum of squares of these mean values (see Eq. 3). This acquires a better representation of the garment fit at group level and is less sensitive to outliers in individual participant pressure measurements.

$\mathrm{RMS}_{\text {group }}=\sqrt{\frac{\sum_{j=1}^{m}\left(y a_{j}\right)^{2}}{m}}$,

where $m$ is the total number of participants and where the mean difference between participants' measured pressure and model calculated pressure $\left(y a_{j}\right)$ was calculated using

$y a_{j}=\frac{\left(\sum_{i=1}^{n} P a_{i}-P b_{i}\right)}{n}$.

All measurements of length were defined from the ankle malleolus relative to the length of the leg which for this study, was defined as the shortest distance between the ankle malleolus and the greater trochanter. The peak pressure and pressure gradient data used for analysis, and subsequently reported in the results, were derived from the RMS method. A one-way repeated measures ANOVA was conducted to examine peak pressure and pressure gradient in the left and the right legs of the participants in the three garment conditions. Significant effects were further analysed using a Bonferroni post hoc test. Paired samples t tests were conducted to assess for differences of peak pressure and pressure gradient between legs of each garment condition. Effect sizes were calculated as partial eta squared $\left(\eta p^{2}\right)$ and interpreted as $0.01=$ small, $0.06=$ medium and $0.14=$ large [25]. Data are presented as mean and standard deviation (mean \pm SD), unless otherwise stated. A significance level of $P<0.05$ was applied throughout.

\section{Results}

\subsection{Root mean squared difference}

The root mean squared differences (Eq. 3) between predicted and actual pressures in the left and right leg, respectively, were: control garment, 2.1 and 2.1; asymmetrical garment, 2.0 and 2.5; symmetrical garment, 2.1 and 2.1 (all $\mathrm{mmHg}$ ). The inter-individual root mean squared differences (Eq. 2) ranged from: control garment, 1.5 to 3.5 ; asymmetrical garment, 1.2 to 6.3 ; symmetrical garment, 1.5 to 4.5 (all $\mathrm{mmHg})$.

\subsection{Pressure gradients between garment conditions}

\subsubsection{Left leg}

There was a difference in pressure gradient between garments for the left leg (main effect condition $[F(2$, $\left.18)=79.527, P=0.001 \eta p^{2}=0.898\right]$, Table 1$)$. The pressure gradient was shallower in the control and asymmetrical garments than in the symmetrical garment (pairwise comparison, $P=0.001$ in both instances). As intended the pressure gradient in the left leg of the asymmetrical garment was the same as in the control garment (pairwise comparison, $P=1.000)$, (Table 1).

\subsubsection{Right leg}

There was a difference in pressure gradient between garments for the right leg (main effect condition $[F(2$, $\left.18)=89.661, P=0.001 \eta p^{2}=0.909\right]$, Table 1$)$. The pressure gradient was shallower in the control garment than in both the asymmetrical and symmetrical garments (pairwise 
Table 1 Pressure gradient in the left and right legs in the control, asymmetrical and symmetrical compression garment conditions (mean $\pm \mathrm{SD}, n=10)$

\begin{tabular}{lcc}
\hline & Left $(\mathrm{mmHg})$ & Right $(\mathrm{mmHg})$ \\
\hline Control & $-8.9 \pm 3.5$ & $-7.4 \pm 3.0$ \\
Asymmetrical & $-7.8 \pm 3.9$ & $-21.9 \pm 3.2^{*},{ }^{\dagger}$ \\
Symmetrical & $-25.0 \pm 4.1^{\dagger}$ & $-22.3 \pm 3.6^{\dagger}$ \\
\hline
\end{tabular}

*Significantly different between legs

${ }^{\dagger}$ Significantly different to control garment $(P<0.05)$

comparison, $P=0.001$ in both instances). As intended the pressure gradient in the right leg of the asymmetrical garment was the same as in the symmetrical garment (pairwise comparison, $P=1.000$ ), (Table 1 ).

\subsection{Peak pressure between garment conditions}

\subsubsection{Left leg}

There was a difference in peak pressure between garments for the left leg (main effect condition $[F(2,18)=115.299$, $\left.P=0.001 \eta p^{2}=0.933\right]$, Table 2). The peak pressure was lower in the control and asymmetrical garments than in the symmetrical garment (pairwise comparison, $P=0.001$ in both instances). As intended the peak pressure in the left leg of the asymmetrical garment was the same as in the control garment (pairwise comparison, $P=1.000$ ), (Table 2).

\subsubsection{Right leg}

There was a difference in peak pressure between garments for the right leg (main effect condition $[F(2,18)=111.708$, $\left.P=0.001 \eta p^{2}=0.925\right]$, Table 2). The peak pressure was lower in the control garment than in both the asymmetrical and symmetrical garments (pairwise comparison, $P=0.001$ in both instances). As intended the peak pressure in the right leg of the asymmetrical garment was the same as in the symmetrical garment (pairwise comparison, $P=1.000$ ), (Table 2).

Table 2 Peak pressure at the ankle in the left and right legs in the control, asymmetrical, symmetrical and uniform compression garment conditions (mean $\pm \mathrm{SD}, n=10$ )

\begin{tabular}{lcc}
\hline & Left $(\mathrm{mmHg})$ & Right $(\mathrm{mmHg})$ \\
\hline Control & $13.5 \pm 2.3$ & $12.9 \pm 2.6$ \\
Asymmetrical & $12.7 \pm 2.5$ & $26.3 \pm 3.4^{*}{ }^{\dagger}$ \\
Symmetrical & $27.7 \pm 2.2^{\dagger}$ & $27.5 \pm 1.6^{\dagger}$ \\
\hline
\end{tabular}

*Significantly different between legs

${ }^{\dagger}$ Significantly different to control garment $(P<0.05)$

\subsection{Within garment between leg pressure gradient differences}

There was a difference in pressure gradient between legs in the asymmetrical garment $[t(9)=14.068, P=0.001]$ and no differences in the control and symmetrical garments $[t(9)=-1.324, P=0.218]$ and $[t(9)=-1.975, P=0.080$, respectively].

\subsection{Within garment between leg peak pressure differences}

There was a difference in peak pressure between legs in the asymmetrical garment $[t(9)=-23.141, P=0.001]$ and no differences in the control and symmetrical garments $[t(9)=0.442, P=0.669]$ and $[t(9)=0.262, P=0.799$, respectively].

\subsection{Elicited pressures within clinical standards}

As intended, for the control garment and the left leg of the asymmetrical garment, elicited pressure was below Class one of clinical compression standards over all of the legs. For the right leg of the asymmetrical garment, 5, 32, 20 and $43 \%$ of elicited pressures were within Class three, Class two, Class one and below clinical compression standards, respectively. For the left leg of the symmetrical garment, 12, 31, 17 and $45 \%$ of elicited pressures were within Class three, Class two, Class one and below clinical compression standards, respectively. Finally, for the right leg of the symmetrical garment, 9, 30, 16 and $45 \%$ of elicited pressures were within Class three, Class two, Class one and below clinical compression standards, respectively, (Fig. 2).

\section{Discussion}

This study shows that it was possible to make made-tomeasure compression garments that elicit pressures within and below clinical standards. Furthermore, it was shown that pressures and gradients can be replicated within and between participants' legs, and between separate compression garments. The control over elicited pressure was evidenced by peak pressure of $27.7 \pm 2.2 \mathrm{mmHg}$ and $27.5 \pm 1.6 \mathrm{mmHg}$ for the symmetrical garment in the left and right legs of participants (within Class three of clinical compression standards), while for the control garment, the corresponding values were $13.5 \pm 2.3 \mathrm{mmHg}$ and $12.9 \pm 2.6 \mathrm{mmHg}$ (below clinical compression standards). The use of individual 3D scans ensured that made-to-measure compression garments consistently elicited prescribed pressure profiles in participants' legs and between different garment conditions. Therefore, no differences in peak pressure or pressure gradient 
Fig. 2 Pooled data presenting pressure profiles for the left and right legs in the a control, $\mathbf{b}$ asymmetrical and $\mathbf{c}$ symmetrical compression garment conditions. Class one $(14-17 \mathrm{mmHg}$ ) clinical compression threshold indicated by light grey shading, Class two $(18-24 \mathrm{mmHg})$ clinical compression threshold indicated by medium grey shading and Class three $(25-35 \mathrm{mmHg}$ ) clinical compression threshold indicated by dark grey shading (BS-6612; 1985). The dashed trendline corresponds to the left leg pressure gradient and the filled trendline corresponds to the right leg pressure gradient

were found between legs in the control and symmetrical garment conditions $(P<0.05)$. Many compression garment studies have either not measured the pressure elicited by a garment [13, 26-29] or they have relied on pressures stated by the manufacturer $[7,14,30]$. As such, it is difficult, if not impossible, to link a particular garment pressure or profile to a particular performance or recovery outcome. In the current study, peak pressures at the ankle for the symmetrical garment were within Class three of the clinical compression standards and within clinical standards over 55 to $60 \%$ of the leg length. Similarly, peak pressures at the ankle for the control garment were below clinical compression standards for both legs. Hill et al. [31] showed that medical grade II compression stockings, which elicited pressures of $24.3 \pm 3.7 \mathrm{mmHg}$ and $14.8 \pm 2.2 \mathrm{mmHg}$ at the calf and thigh, respectively, improved maximal voluntary contractions and counter-movement jump recovery $(P<0.05)$. These peak pressures are similar to those elicited by the symmetrical garment in the current study.

The current study sought to develop compression garments that provided the same fitting between a participants' legs, as well as between participants within the same garment condition. This was achieved as evidenced by the pressure gradients between legs showing absolute differences of only 1.4 and $2.7 \mathrm{mmHg}$ for the control and symmetrical garments, respectively, and the corresponding absolute differences in peak pressure at the ankle were also small (0.6 and $0.2 \mathrm{mmHg}$, respectively). Previous research has found size and symmetry differences between legs, in male cyclists of up to $2 \%$ at the calf and knee [21]. As commercially available compression garments are made assuming the geometry of both legs is the same, this may lead to an inconsistent fit on an individuals' legs. The studies that have measured the pressure elicited by a compression garment, only measured pressure on one leg $[20,32,33]$ or did not report pressure data between legs $[31,34,35]$, which presents a challenge when comparing the current results to previous research. However, any effect of leg asymmetry and between participant differences was not a factor in the current study, as the made-to-measure compression garments were individually designed and made for each participant. The RMS difference for the fit of each garment showed a good fit between legs within each garment condition (control: $<2.1 \mathrm{mmHg}$; symmetrical $<2.5 \mathrm{mmHg}$ ) as well as between participants
A

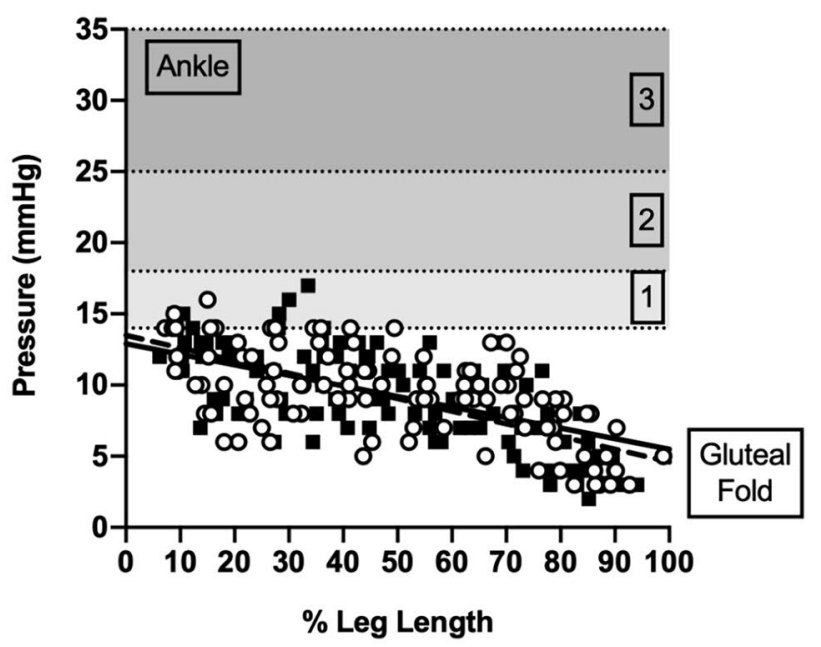

B

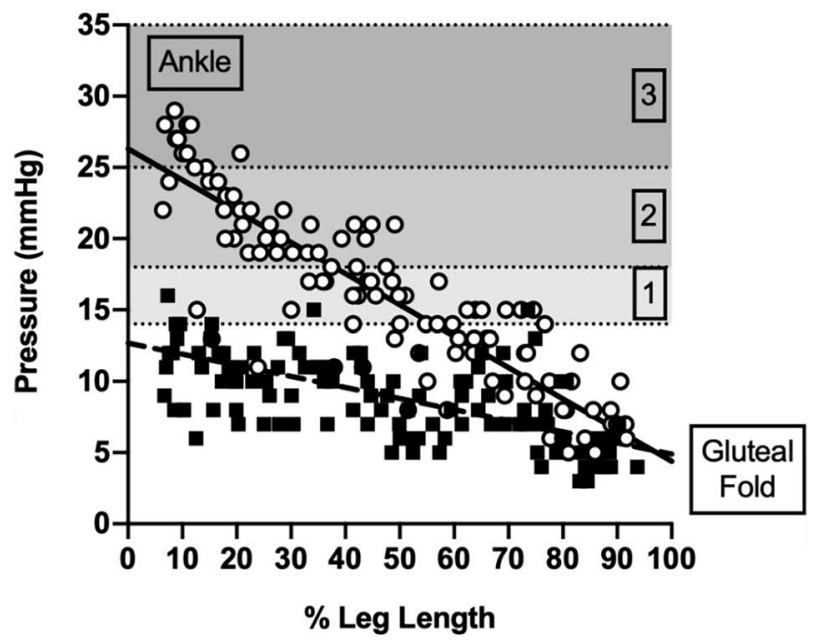

C

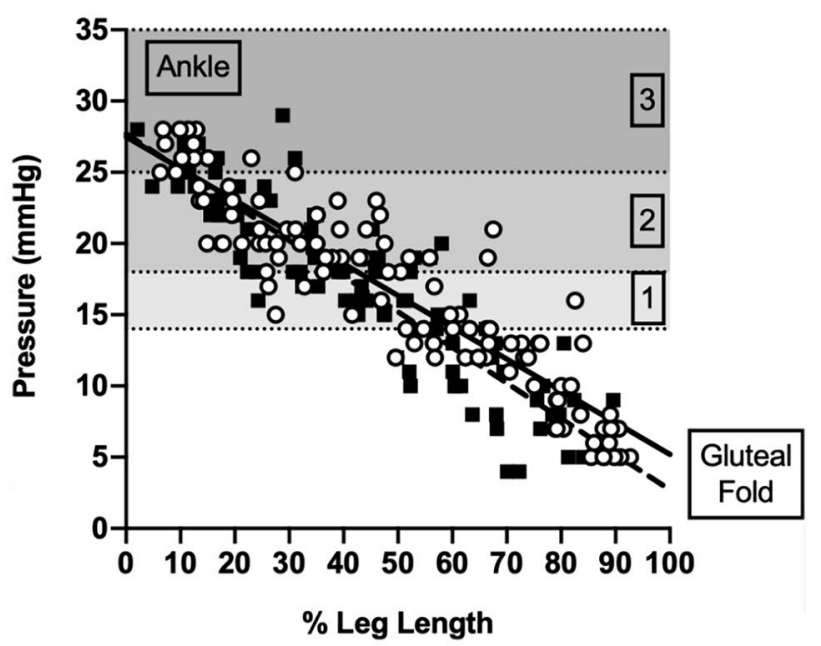


(control: 1.5-3.5 mmHg; symmetrical: $1.2-6.3 \mathrm{mmHg}$; asymmetrical $1.5-4.5 \mathrm{mmHg}$ ). These results demonstrate that made-to-measure compression garments can compensate for leg asymmetry and participant-specific differences.

Compression garment manufacturers claim that their garments elicit graduated compression, whereby the highest pressure is located at the distal end of the garment and reduces proximally [1]. Therefore, peak pressure would be located at the ankle when wearing lower body compression garments. It has been found that in undersized, recommended sized and oversized commercially available compression tights, peak pressure was located at the maximal circumference of the calf [20]. As a result, a linear pressure gradient was not present within the examined compression garments. This means that standard-sized commercially available compression garments may not elicit a pressure gradient suitable to aid venous flow. In the current study, as intended, a linear pressure gradient was evident within the symmetrical garment as peak pressure was located at the ankle and this reduced linearly towards the gluteal fold. In clinical practice, when wearing a compression garment, it is recommended that pressure at the thigh is $40 \%$ lower than at the ankle [36]. This pressure gradient is proposed to increase arterial pressure and subsequently elevate venous return, thus reducing venous pooling in the lower extremities [36]. In the current study, pressure in the symmetrical garment reduced by $>80 \%$ at the gluteal fold from peak pressure at the ankle. However, there is no evidence to suggest that the pressure gradients used in clinical practice provide performance or recovery benefits when applied in healthy sports participants. In addition, while a range of elicited pressures have been reported at different leg locations for commercially available compression garments (19.0 to $30.0 \mathrm{mmHg}$ at the ankle, 17.6 to $25.0 \mathrm{mmHg}$ at the calf and 9.1 to $18.0 \mathrm{mmHg}$ at the thigh), typically these pressures have not been measured in participants whilst wearing the compression clothing [37]. An advantage with the made-tomeasure garments used in the current study is that they can be adjusted to elicit different pressures and pressure gradients, which could be useful for future research to determine whether wearing compression garments influences exercise performance and recovery.

At the time the current study was conducted, the Kikuhime pressure monitoring device was an established method for measuring the pressures elicited by compression garments $[23,38]$. Recent research has challenged the validity of the Kikuhime pressure monitoring device and recommended the use of an alternative device (PicoPress) [39]. The study by McManus and colleagues [39] clearly demonstrated that the Kikuhime pressure monitoring device overestimated criterion pressure (as established using the Hohenstein System) by $2.9 \mathrm{mmHg}$ (compared with $0.2 \mathrm{mmHg}$ for the PicoPress pressure monitoring device).
However, in the other two analytical methods of assessment used in the study to compare the pressures derived from the measuring devices with a criterion (that is Bland and Altman's limits of agreement [40] and regression analysis), the Kikuhime pressure monitoring device outperformed the PicoPress device when the measurements were made at the posterior aspect of the calf and the compression garments being worn were tights (Kikuhime vs. PicoPress: Limits of Agreement, \pm 4.2 vs. $\pm 4.9 \mathrm{mmHg}$; Regression slope parameter estimate 0.2 vs. $1.3^{1}$ ). Given: (1) the smaller limits of agreement and slope parameter noted above; (2) that the differences measured in the current study were $>3 \mathrm{mmHg}$; and (3) that all pressure measurements were made along the posterior aspect of the leg as recommended by [39]; it can be concluded that the Kikuhime pressure monitoring device was adequate for making the measurements here. Another recent study [41] noted that the dimensions of the air-filled sensor on a pressure monitoring device may influence the accuracy of the pressure measurement, and the PicoPress has a relatively large sensor area $(50 \mathrm{~mm})$ which may limit its ability to measure pressure accurately on small or curved areas. Conversely, the Kikuhime device has a smaller sensor area $(38 \times 30 \mathrm{~mm})$ which makes it more suitable for measuring pressure on areas with high curvatures that are found on parts of the human leg (e.g. Achilles tendon).

\section{Conclusion}

The current study demonstrated that made-to-measure compression garments can be made to elicit pressures within and below clinical compression standards, and elicit equivalent pressures and pressure gradients in different participants. The results suggest that made-to-measure compression garments could be used within sport to provide an optimum fit between and within individuals using measurements from individuals' bodies rather than generic manufacturer sizing. Further research is warranted to investigate the effect of made-to-measure compression garments on exercise performance and recovery.

Acknowledgements The authors wish to thank the participants for their invaluable contribution to the study. The authors wish to thank Kurio 3D Compression Limited for supplying the compression garments for the study.

Funding This study did not receive any funding.

Availability of data and material Any queries regarding the data used in this study may be directed to the corresponding author.

\footnotetext{
1 The more consistent the value given by the pressure monitoring device as the criterion pressure changes, the closer the parameter estimate is to zero, zero being the ideal.
} 


\section{Declarations}

Conflict of interest The authors declare that they have no conflict of interests. The compression garments used in the current study were provided at no cost by Kurio 3D Compression Ltd as part of an ongoing project evaluating compression wear. There is no formal agreement between the company and the authors.

Ethics approval The study was approved by a University Ethics Committee.

Consent to participate All participants completed an informed consent form after viewing a participant information sheet and given the opportunity to ask any questions.

Consent for publication All authors have agreed to submit the manuscript in its current form for consideration for publication in the Journal of Sports Engineering.

Open Access This article is licensed under a Creative Commons Attribution 4.0 International License, which permits use, sharing, adaptation, distribution and reproduction in any medium or format, as long as you give appropriate credit to the original author(s) and the source, provide a link to the Creative Commons licence, and indicate if changes were made. The images or other third party material in this article are included in the article's Creative Commons licence, unless indicated otherwise in a credit line to the material. If material is not included in the article's Creative Commons licence and your intended use is not permitted by statutory regulation or exceeds the permitted use, you will need to obtain permission directly from the copyright holder. To view a copy of this licence, visit http://creativecommons.org/licenses/by/4.0/.

\section{References}

1. MacRae BA, Cotter JD, Laing RM (2011) Compression garments and exercise: garment considerations, physiology and performance. Sport Med 41:815-843. https://doi.org/10.2165/11591 420-000000000-00000

2. Doan BK, Kwon Y, Newton RU et al (2003) Evaluation of a lowerbody compression garment. J Sport Sci 21:601-610. https://doi. org/10.1080/0264041031000101971

3. Todd M (2015) Compression hosiery choices for managing chronic oedema. Br J Community Nurs 20:318-320. https://doi. org/10.12968/bjen.2015.20.7.318

4. Xiong Y, Tao X (2018) Compression garments for medical therapy and sports. Polymers (Basel) 10:663. https://doi.org/10.3390/ polym 10060663

5. Yang C, Xu Y, Yang Y et al (2020) Effectiveness of using compression garments in winter racing sports: a narrative review. Front Physiol 11:1-10. https://doi.org/10.3389/fphys.2020.00970

6. Agu O, Hamilton G, Baker D (1999) Graduated compression stockings in the prevention of venous thromboembolism. Br J Surg 86:992-1004. https://doi.org/10.1046/j.1365-2168.1999.01195.x

7. Kim J, Kim J, Lee J (2017) Effect of compression garments on delayed-onset muscle soreness and blood inflammatory markers after eccentric exercise: a randomized controlled trial. J Exerc Rehabil 13:541-545. https://doi.org/10.12965/jer.1735088.554

8. Rugg S, Sternlicht E (2013) The effect of graduated compression tights, compared with running shorts, on counter movement jump performance before and after submaximal running. J Strength Cond Res 27:1067-1073. https://doi.org/10.1519/JSC.0b013 e3182610956
9. Kraemer W, Flanagan S, Comstock B et al (2010) Whole body compression garment on markers of recovery after an intense whole body resistance training workout in men. J Strength Cond Res 34:804-814

10. Broatch JR, Bishop D, Halson S (2017) Lower-limb sports compression garments improve exercise performance and muscle blood flow during repeated-sprint cycling. Int J Sports Physiol Perform 13:882-890

11. Sear J, Hoare T, Scanlan A et al (2010) The effects of whole-body compression garments on prolonged high-intensity intermittent exercise. J Strength Cond Res 24:1901-1910

12. Kemmler W, von Stengel S, Köckritz C et al (2009) Effect of compression stockings on running performance in men runners. J Strength Cond Res 23:101-105

13. Winke M, Williamson S (2017) Comparison of a pneumatic compression device to a compression garment during recovery from DOMS. Med Sci Sport Exerc 11:375-383

14. Govus AD, Andersson EP, Shannon OM et al (2018) Commercially available compression garments or electrical stimulation do not enhance recovery following a sprint competition in elite cross-country skiers. Eur J Sport Sci 18:1299-1308. https://doi. org/10.1080/17461391.2018.1484521

15. Struhár I, Kumstát M, Králová DM (2018) Effect of compression garments on physiological responses after uphill running. J Hum Kinet 61:119-129. https://doi.org/10.1515/hukin-2017-0136

16. Del Coso J, Areces F, Salinero JJ et al (2014) Compression stockings do not improve muscular performance during a half-ironman triathlon race. Eur J Appl Physiol 114:587-595. https://doi.org/ 10.1007/s00421-013-2789-2

17. Stickford ASL, Chapman RF, Johnston JD, Stager JM (2015) Lower-leg compression, running mechanics, and economy in trained distance runners. Int J Sports Physiol Perform 10:76-83. https://doi.org/10.1123/ijspp.2014-0003

18. Sperlich B, Born DP, Kaskinoro K et al (2013) Squeezing the muscle: compression clothing and muscle metabolism during recovery from high intensity exercise. PLoS ONE. https://doi. org/10.1371/journal.pone.0060923

19. Partsch H, Partsch B, Braun W (2006) Interface pressure and stiffness of ready made compression stockings: comparison of in vivo and in vitro measurements. J Vasc Surg 44:809-814. https://doi. org/10.1016/j.jvs.2006.06.024

20. Brophy-Williams N, Driller MW, Shing CM et al (2015) Confounding compression: the effects of posture, sizing and garment type on measured interface pressure in sports compression clothing. J Sports Sci 33:1403-1410. https://doi.org/10.1080/02640 414.2014.990489

21. Rauter S, Vodicar J, Simenko J (2017) Body asymmetries in young male road cyclists. Int J Morphol 35:907-912. https://doi.org/10. 4067/S0717-95022017000300018

22. Liu R, Guo X, Lao TT, Little T (2017) A critical review on compression textiles for compression therapy: textile-based compression interventions for chronic venous insufficiency. Text Res J 87:1121-1141. https://doi.org/10.1177/0040517516646041

23. Brophy-Williams N, Driller MW, Halson SL et al (2014) Evaluating the Kikuhime pressure monitor for use with sports compression clothing. Sport Eng 17:55-60. https://doi.org/10.1007/ s12283-013-0125-z

24. Corana A, Marchesi M, Martini C, Ridella S (1987) Minimizing multimodal functions of continuous variables with the "simulated annealing" algorithm. ACM Trans Math Softw 13:262-280. https://doi.org/10.1145/29380.29864

25. Cohen J (1988) Statistical power analysis for the behavioural sciences, 2nd edn. Routledge Academic, New York

26. Shimokochi Y, Kuwano S, Yamaguchi T et al (2017) Effects of wearing a compression garment during night sleep on recovery 
from high-intensity eccentric-concentric quadriceps muscle fatigue. J Strength Cond Res 31:2816-2824

27. Cerqueira MS, Santos Borges L, dos Santos Rocha JA et al (2015) Twelve hours of a compression sleeve is not enough to improve the muscle recovery of an exercise-damaged upper arm. Apunt Med l'Esport 50:23-28. https://doi.org/10.1016/j.apunts.2014.07. 003

28. Pereira M, Bottaro M, Brown L et al (2014) The effects of graduated compression sleeves on muscle performance: a randomised controlled trial. Int J Sport Sci Coach 9:985-992. https://doi.org/ 10.1260/1747-9541.9.5.985

29. Duffield R, Cannon J, King M (2010) The effects of compression garments on recovery of muscle performance following high-intensity sprint and plyometric exercise. J Sci Med Sport 13:136-140. https://doi.org/10.1016/j.jsams.2008.10.006

30. Heiss R, Hotfiel T, Kellermann M et al (2018) Effect of compression garments on the development of edema and soreness in delayed-onset muscle soreness (DOMS). J Sport Sci Med 17:392-401

31. Hill J, Howatson G, Van Someren K et al (2017) The effects of compression-garment pressure on recovery after strenuous exercise. Int J Sports Physiol Perform 12:1078-1084. https://doi.org/ 10.1123/ijspp.2016-0380

32. Brophy-Williams N, Driller MW, Kitic CM et al (2017) Effect of compression socks worn between repeated maximal running bouts. Int J Sports Physiol Perform 12:621-627. https://doi.org/ 10.1123/ijspp.2016-0162

33. Hamlin M, Mitchell C, Ward F et al (2012) Effect of compression garments on short term recovery of repeated sprint and 3-km running performance in rugby union players. J Strength Cond Res 26:2975-2982

34. Born DP, Holmberg HC, Goernert F, Sperlich B (2014) A novel compression garment with adhesive silicone stripes improves repeated sprint performance-a multi-experimental approach on the underlying mechanisms. BMC Sports Sci Med Rehabil. https://doi.org/10.1186/2052-1847-6-21

35. Scanlan AT, Dascombe BJ, Reaburn PRJ, Osborne M (2008) The effects of wearing lower-body compression garments during endurance cycling. Int J Sports Physiol Perform 3:424-438. https://doi.org/10.1123/ijspp.3.4.424

36. Oğlakcıoğlu N, Marmaralı A (2014) An approach for measuring pressure characteristics of medical compression stockings. AATCC J Res 1:20-27. https://doi.org/10.14504/ajr.1.2.3

37. Hill JA, Howatson G, van Someren KA et al (2015) The variation in pressures exerted by commercially available compression garments. Sport Eng 18:115-121. https://doi.org/10.1007/ s12283-015-0170-x

38. Van den Kerckhove E, Fieuws S, Massagé P et al (2007) Reproducibility of repeated measurements with the Kikuhime pressure sensor under pressure garments in burn scar treatment. Burns 33:572-578. https://doi.org/10.1016/j.burns.2006.08.032

39. McManus C, Venkatraman PD, Sandercock G (2020) The measurement of interface pressure applied by sports compression garments: a comparative study of two portable devices. Meas J Int Meas Confed 156:107592. https://doi.org/10.1016/j.measurement. 2020.107592

40. Bland JM, Altman DG (1986) Statistical methods for assessing agreement between two methods of clinical measurement. Lancet $1: 307-310$

41. Nandasiri GK, Shahidi AM, Dias T (2020) Study of three interface pressure measurement systems used in the treatment of venous disease. Sensors 20:5777

Publisher's Note Springer Nature remains neutral with regard to jurisdictional claims in published maps and institutional affiliations. 